\title{
Terapia de activación conductual para la depresión: aplicación a un paciente con esquizofrenia paranoide
}

\author{
Rafael Romero Gamero \\ Hospital Universitario Virgen Macarena, Sevilla
}

\author{
Silvia Poves Oñate \\ Hospital Virgen de la Merced, Osuna (Sevilla)
}

\author{
Nicolás Vucinovich \\ Hospital Universitario Virgen del Rocío, Sevilla
}

\section{RESUMEN}

En el presente trabajo se expone el tratamiento de un trastorno depresivo en un individuo con un diagnóstico de esquizofrenia. El paciente ha recibido un tratamiento basado en los principios de la Terapia de Activación Conductual durante diez semanas. Las medidas utilizadas (CDSS, PANSS, EROS) indican una mejoría significativa de la sintomatología depresiva, dejando de cumplir criterios de episodio depresivo, mejoría en la sintomatología, tanto psicótica como general, y un aumento en los niveles del reforzamiento positivo percibido. Estos resultados se han mantenido en el seguimiento de diez semanas. Estudios futuros deberían de confirmar estos datos iniciales.

Palabras clave: activación conductual, depresión, psicosis

\section{ABSTRACT}

Treatment for a man with depressive disorder and diagnosed with paranoid schizophrenia is set out. The intervention was based on the Behavioral Activation Therapy for ten weeks. Pretreatment measures (CDSS, PANSS, EROS) show a significant improvement. Depressive disorder was in remission and we find a remarkable improvement in symptoms, psychotic as well as general symptomatology. We also find an increased levels of perceived positive reinforcement. Improvement has been remained until ten weeks monitoring. The good outcomes obtained in this case support the model proposed by Behavioral Activation to reduce depression in a patient with schizophrenia. Next reserch must confirm this inicial data.

Keywords: Behavioral activation, depression, psychosis 
La existencia de tratamientos psicológicos de demostrada eficacia y efectividad para los trastornos del espectro afectivo es un hecho incuestionable. Entre ellos destacan la Terapia Cognitivo-Conductual (Elkin et al., 1989; NICE, 2004; Thase et al., 1997) y la Psicoterapia Interpersonal (Elkin et al., 1989). Sin embargo, los resultados de los últimos estudios sobre los componentes eficaces de la Terapia Cognitiva, donde se ha observado que la eficacia del componente conductual es equivalente a todo el tratamiento completo, defiende el uso de éstas estrategias a la hora de abordar la sintomatología depresiva (Dimidjian, Hollon, Dobson, Schmeling, Kohlenberg, \& Addis, 2006; Jacobson et al., 1996). Esto ha dando lugar al desarrollo de la denominada Terapia de Activación Conductual para la Depresión (Jacobson, Martell, \& Dimidjian, 2001).

Éste nuevo enfoque de abordar los problemas psicológicos presenta una serie de características que lo diferencia del enfoque tradicional del tratamiento cognitivo-conductual (Pérez Álvarez, 2006):

1) Se destaca el papel del contexto donde se desenvuelve del sujeto.

2) Se prioriza que el paciente esté en contacto con actividades que puedan ser reforzadas en su contexto.

3) Se modifica el ambiente, no el pensamiento ni las emociones.

4) El foco de atención psicopatológica es impedir la evitación conductual.

5) La persona es entendida en el ámbito contextual e idiográfico.

6) Se enfatizan las contingencias naturales de reforzamiento.
Esta nueva perspectiva ha dado lugar al desarrollo de manuales de tratamiento como el de la Activación Conductual (AC) de Martell, Addis y Jacobson (2001) y el de Lejuez, Hopko y Hopko (2001), mas breve y parsimonioso denominado concretamente Terapia Breve de Activación Conductual de la Depresión (TACD), cuya meta radica en guiar la actividad de los clientes hacia unos objetivos concretos. Por esa razón, la principal técnica que se propone es la programación de actividades y la monitorización a través de registros de actividades. A nivel teórico se basa en la relevancia del reforzamiento positivo y la de la ley de igualación de Hernstein (1970) que indica que si los reforzadores obtenidos por presentar conductas definidas como depresivas (por ejemplo, atención por parte de familiares y amigos, evitación de actividades consideradas como desagradables, etc.) son superiores a los inconvenientes obtenidos de éstas (por ejemplo, la preocupación y queja de amigos y familiares, no realización de actividades gratificantes, etc.) la situación se mantendrá. En consecuencia, sólo haciendo que los beneficios de la conducta depresiva disminuyan existirán posibilidades de que cambie la situación. Por eso, la TACD destaca como relevante que no se obtenga beneficio por actuar depresivamente, y que, en cambio, se busquen y se ofrezcan nuevos reforzadores al sujeto cuando actúe de forma no depresiva. Igualmente, en la TACD, por el tipo de implementación de la terapia, la colaboración entre paciente y terapeuta es imprescindible buscando que el sujeto se responsabilice del cambio participando activamente en el curso 
de la terapia, en especial, en el trabajo entre sesiones (Lejuez et al., 2001). Además, al igual que en el protocolo de la AC, al explicar al paciente la lógica y el curso de la terapia, se le comenta lo relevante que es para el cambio de sus sentimientos y pensamientos que primero inicie ciertas actividades y que, progresivamente, esas acciones resultarán cada vez más fáciles de realizar. Respecto a la estructuración de la terapia, hay que mencionar que aunque al principio se programen sesiones de una hora aproximadamente esta duración sólo es necesaria en las primeras fases de tratamiento, reduciéndose luego a sesiones de 15 y 30 minutos. En cuanto a la periodicidad de sesiones el terapeuta puede ver al paciente tres o más veces por semana, para espaciar luego las sesiones a dos o una vez por semana (Barraca, 2009).

Los resultados empíricos sobre la eficacia de los modelos basados en la activación conductual defienden su uso en cuadros depresivos graves (Dimidjian et al, 2006). Existe, asimismo, aval de su eficacia en pacientes deprimidos hospitalizados siendo muy superior al tratamiento de apoyo psicológico (Hopko et al., 2001). Su aplicación se está extendiendo a otras situaciones como trastornos ansiosos-depresivos (Hopko, Robertson, \& Lejuez, 2006), en consumidores de drogas con síntomas depresivos (Daughters et al., 2008), en enfermos de cáncer (Hopko, Bell, Armento, Hunt, \& Lejuez, 2005), trastornos limite de la personalidad con ideación autolítica (Hopko, Sanchez, Hopko, Dvir, \& Lejuez, 2003 ) o en adolescentes (Ruggiero, Morris, Hopko, \& Lejuez, 2007).
Por otro lado, los pacientes que reciben un diagnóstico de esquizofrenia deben hacer frente a una serie de cambios que suponen una ruptura con el estilo de vida llevado hasta esos momentos: seguir un tratamiento estricto, una reducción o eliminación de la actividad laboral, limitaciones inducidas por la sintomatología psicótica, tanto negativa como positiva, que le lleva al abandono y evitación de situaciones que suponen una gratificación demorada. La propia dinámica familiar, tanto previa como posterior al diagnóstico, puede determinar en gran medida la afectación en el funcionamiento personal y social que los síntomas pueden provocar. El riesgo de suicidio no es tema a minimizar en éstos casos (Meltzer \& Baldessarini, 2003). Todo éste contexto al que se ve avocada puede llevar a la persona a un estado depresivo de diversa intensidad donde el abandono de actividades previamente gratificantes se puede encontrar en la base del mencionado estado.

Con el presente artículo los autores desean ampliar el uso del enfoque de la Activación Conductual al tratamiento de la sintomatología depresiva en casos de patología mental grave presentando el de un paciente con diagnóstico de esquizofrenia paranoide.

\section{Método}

\section{Participante}

A. es un hombre de 45 años residente en una zona rural y con un diagnóstico de esquizofrenia paranoide. Se encuentra en tratamiento desde el año 1995. Actualmente su sintomatología alucinatorio-delirante se encuentra contenida en gran parte con los tratamientos 
de medicación, psicológicos y de enfermería recibidos.

El paciente cuenta con estudios primarios y ha trabajado en el sector agrícola por periodos. Se describe como tímido y con dificultad para establecer nuevas relaciones. El inicio de su trastorno psicótico fue paulatino, empezando a reducir sus contactos sociales, con un aumento de suspicacia y temor a sufrir insultos y agresiones físicas, refiere que percibía a su alrededor unas voces que le indicaban que tuviera cuidado con las personas. El paciente convive con la madre. Tiene dos hermanos, todos mayores que él con los que mantenía unas adecuadas pero superficiales relaciones.

Sin embargo, durante el último año refiere importantes sentimientos de tristeza, desesperanza, autodepreciación y de culpa y una reducción de sus pocas aficiones: cría de aves, paseos, quedar con viejas amistades o escuchar la radio. Se observa una elevación de sus niveles de ansiedad previos. Ha ido percibiendo un incremento de su nivel de suspicacia y temor a ser agredido cuando transita por su localidad con el riesgo consecuente de descompensación psicótica.

Tras la exploración inicial se observa que el inicio de la sintomatología afectiva referida se relacionaba con el empeoramiento físico de la madre por dolencias de tipo reumatológicas. A. intentaba pasar más tiempo junto a ella con el objetivo de cuidarla a pesar de que ella disponía de la suficiente autonomía. Con el tiempo, el paciente, fue abandonando actividades gratificantes comenzando a presentar una disminución de su estado de ánimo.

\section{Instrumentos}

Se ha seleccionado las siguientes pruebas debido a la idoneidad para la valoración de la sintomatología depresiva así como la influencia que pudiera tener nuestra intervención en otras áreas de la psicopatología.

La Escala de Depresión de Calgary (Calgary Depression Scale for Schizophrenia, CDSS) es un instrumento desarrollado específicamente para valorar el nivel de depresión en la esquizofrenia, tanto en fase aguda como en los estados crónicos (Addington, Addington, \& Schissel, 1990). Se compone de 9 ítems, centrada fundamentalmente en los síntomas cognitivos de la depresión, con una graduación de intensidad sintomática de 4 puntos (ausente, leve, moderado, grave). El rango de puntuación es de 0-27. No existen criterios para categorizar la gravedad de la depresión, considerándose una medida continua de intensidad sintomática. Una puntuación igual o inferior de 5 supone ausencia de depresión.

La Escala de Síndrome Positivoy Negativo(The Positive and Negative Síndrome Scale, PANSS) fue desarrollada por Kay, Fiszbein y Opler en 1987 y está basada en la Brief Psychiatric Rating Scale (BPRS) de Overall y Gorham (1962). Consta de 30 síntomas que se puntúan de 1 (ausente) a 7 (extremo). Siete síntomas evalúan el síndrome positivo (PANSS-P) de la esquizofrenia, otros siete los del síndrome negativo (PANSS-N) y los dieciséis restantes constituyen la escala de psicopatología general (PANSS-PG). El estudio de la validación española de la PANSS (Peralta \& Cuesta, 1994) señaló una fiabilidad interobservador de 0,71 y 0,80 , para las escalas positiva y negativa, respectivamente; en la escala de psi- 
copatología general, el acuerdo fue moderado. La consistencia interna de la escala positiva resultó moderada $(0,62)$ y la de la escala negativa elevada $(0,92)$; la escala de psicopatología general presentó una consistencia interna modesta $(0,55)$ (Peralta \& Cuesta, 1994). La validez de criterio se mostró elevada en las escalas positiva $(r=0,70)$ y negativa $(r=0,81)$.

Environmental Reward Observation Scale: EROS (Armento \& Hopko, 2007) consiste en una escala breve de 10 ítems que se ha desarrollado para obtener una autoevaluación objetiva del grado en que el entorno resulta reforzante para el sujeto. Una puntuación alta se relaciona con un aumento de las conductas y del afecto positivo como consecuencia de experiencias reforzantes desde el medio. Este instrumento posee una buena consistencia interna (alfa de Cronbach $=0,85$ ) y una buena fiabilidad testretest $\left(r_{x x}=0,85\right)$. Respecto a la validez de constructo la escala presenta correlaciones con el Pleasant Events Schedule y el BDI). Existe una adaptación española realizada por Barraca \& Pérez-Álvarez (2010). Las propiedades psicométricas de la adaptación española revelan que, de forma semejante a lo que sucede con la escala original en inglés, la versión española del EROS muestras unos buenos niveles de fiabilidad y validez.

\section{Procedimiento}

El paciente es derivado para abordaje de su sintomatología psicótica residual y las limitaciones funcionales actuales. Desde el primer momento se observó un importante nivel de sintomatología de tipo depresivo por lo que se propuso su abordaje específico desde el prin- cipio y que estuviera en sintonía con el tratamiento global que iba a recibir en la unidad de rehabilitación. Tras la primera valoración se consideró que el paciente cumplía criterios, según la CIE-10 (Organización Mundial de la Salud, 1992), de episodio depresivo mayor de intensidad moderada (F32.1).

El análisis funcional concluyó que un tratamiento basado en los principios de la activación conductual sería el más idóneo. Se ha considerado que la disminución del refuerzo positivo ocupa un lugar explicativo básico de la sintomatología presentada por lo que se decidió decantarse por un procedimiento basado en el enfoque desarrollado por Lejuez et al (2001), adaptándolo a las características propias del paciente. En éste particular, el autorregistro que se solicita desde éste modelo no ha podido realizarse al referir desde un principio dificultades de lecto-escritura unido, probablemente, a cierta desconfianza inicial propia de su cuadro psicopatológico. Hemos primado, en éste caso, el fomento de una buena alianza terapéutica realizándose conjuntamente ésta actividad en consulta.

La intervención consistió en 10 semanas con un total de 20 sesiones. Se programaron dos sesiones por semana. La primera, los lunes, de 30-40 minutos de duración. La segunda sesión semanal se realizaba los jueves o viernes, de 20-30 minutos. Durante las dos primeras semanas se dedicó a la valoración del caso, estudio psicométrico inicial, presentación del enfoque de la Activación Conductual, conocer su opinión y a consensuar los objetivos. Las ocho semanas restantes consistieron en la ejecución del tratamiento propuesto con el moldeamien- 
to de las actividades. Durante la primera sesión semanal se realizaba la valoración del plan llevado durante la semana anterior y se realizaba la propuesta de nuevas actividades. La segunda sesión semanal se dedicada a resolver las dudas en la ejecución de las actividades y se realizaba la valoración psicométrica semanal.

Tras la valoración y análisis funcional inicial, realizados durante las dos semanas primeras se concretaron once actividades que tenían o podían tener un carácter gratificante para el paciente. Para cada actividad se establece una frecuencia y duración ideal y se fue monitorizando semana por semana la consecución del objetivo, p. ej.: pasear por las mañanas una hora cinco veces a la semana. Se espera que al final del tratamiento cada actividad sea cubierta tanto en frecuencia como en duración.

A continuación (Tabla 1) se señalan las actividades seleccionadas en la valoración inicial, semana de inicio, la frecuencia con la duración prevista final para cada una de ellas y la sema- na en que se consigue el objetivo propuesto según frecuencia y duración.

Durante las sesiones se vinculaba el estado de ánimo con la realización de actividades, utilizándose para ello gráficos y registros que iban realizando de forma conjunta terapeuta y paciente. Se señalaba siempre la importancia de mantener y perseverar en su ejecución para conseguir una mejoría paulatina a medio plazo. En la selección de actividades se primó las que ya contaba en su repertorio y había dejado de ejecutar. Se buscaban actividades que habían supuesto una gratificación previa para el paciente. A esto se le sumó las actividades que se programaron dentro de su plan de tratamiento consensuado con él y que consideraba valiosas por su utilidad de cara a mejorar de su estado depresivo, en particular las vinculadas a las relaciones con los demás, aspecto éste que es especialmente valorado por él. Asimismo, se concretaban el nivel de ejecución y dificultad de cada actividad para su implementación paulatina y en correspondencia con la capaci-

Tabla 1

Actividades seleccionadas

\begin{tabular}{|c|c|c|c|c|}
\hline Actividad & Semana de inicio & Frecuencia & Duración & Semana de finalización \\
\hline Asistir a las citas de terapia & $1 \underline{a}$ & 2 veces & $20-40 \mathrm{~min}$. & $1 \underline{a}$ \\
\hline Asistir al taller de cocina & $1 \underline{a}$ & $1 \mathrm{vez}$ & $2 \mathrm{~h}$. & $1 \underline{a}$ \\
\hline Acudir al taller de carpintería & $2 \underline{a}$ & 3 veces & $1 \mathrm{~h}$. & $2 \underline{a}$ \\
\hline Acudir a grupo de salud & $2 \underline{a}$ & $1 \mathrm{vez}$ & sin definir & $3 \underline{a}$ \\
\hline Salidas de tiempo libre & $2 \underline{a}$ & $1 \mathrm{vez}$ & sin definir & $3 \underline{a}$ \\
\hline Acudir al taller de relajación & 3 a & $1 \mathrm{vez}$ & $1 \mathrm{~h}$. & $3 \underline{a}$ \\
\hline Cuidar sus mascotas & $3 \underline{a}$ & 7 veces & $1 \mathrm{~h}$. & $5 \underline{a}$ \\
\hline Practicar la relajación en casa & $4 \underline{a}$ & 7 veces & $30 \mathrm{~min}$. & 6a \\
\hline Escuchar la radio & $5 \underline{a}$ & 5 veces & $1 \mathrm{~h}$. & $7 \underline{a}$ \\
\hline Pasear & 6a & 5 veces & $45 \mathrm{~min}$. & $8 \underline{a}$ \\
\hline Quedar con amigos & $7 \underline{a}$ & 3 veces & sin definir & $8^{\underline{a}}$ \\
\hline
\end{tabular}


dad del paciente en cada momento. P. ej: comenzar paseando 45 minutos 3 veces durante la primera semana e ir incrementándola semanalmente hasta las 5 veces durante 1 hora.

El paciente fue evaluado a las diez semanas de terminar el tratamiento activo. Durante este tiempo el paciente siguió acudiendo al centro continuando con el programa propuesto pero sin recibir supervisión directa del terapeuta. Se programaron citas quincenales de seguimiento clínico y apoyo psicológico. Finalmente comentar que el paciente no ha sufrido ninguna modificación en su medicación durante el periodo que recibió la presente intervención ni uso de medicación antidepresiva, prefiriendo un abordaje psicológico en primer lugar.

\section{Resultados}

A continuación se exponen los resultados respecto a la sintomatología depresiva. Ésta fue valorada semanalmente durante el tiempo que duró el tratamiento así como al seguimiento. En la Figura 1 se muestran los resultados obtenidos durante las 10 semanas de tratamiento.

Los datos en la CDSS demuestran un claro nivel de sintomatología depresiva al comienzo de la intervención confirmando la impresión clínica inicial. Tras la intervención el paciente muestra una puntuación de 4 puntos, inferior al punto de corte de 5 puntos establecido para indicar síndrome depresivo (Addington et al, 1990).

Finalmente, se ofrecen (Tabla 2) los resultados obtenidos al inicio, al final y a las diez semanas de finalizar la intervención.

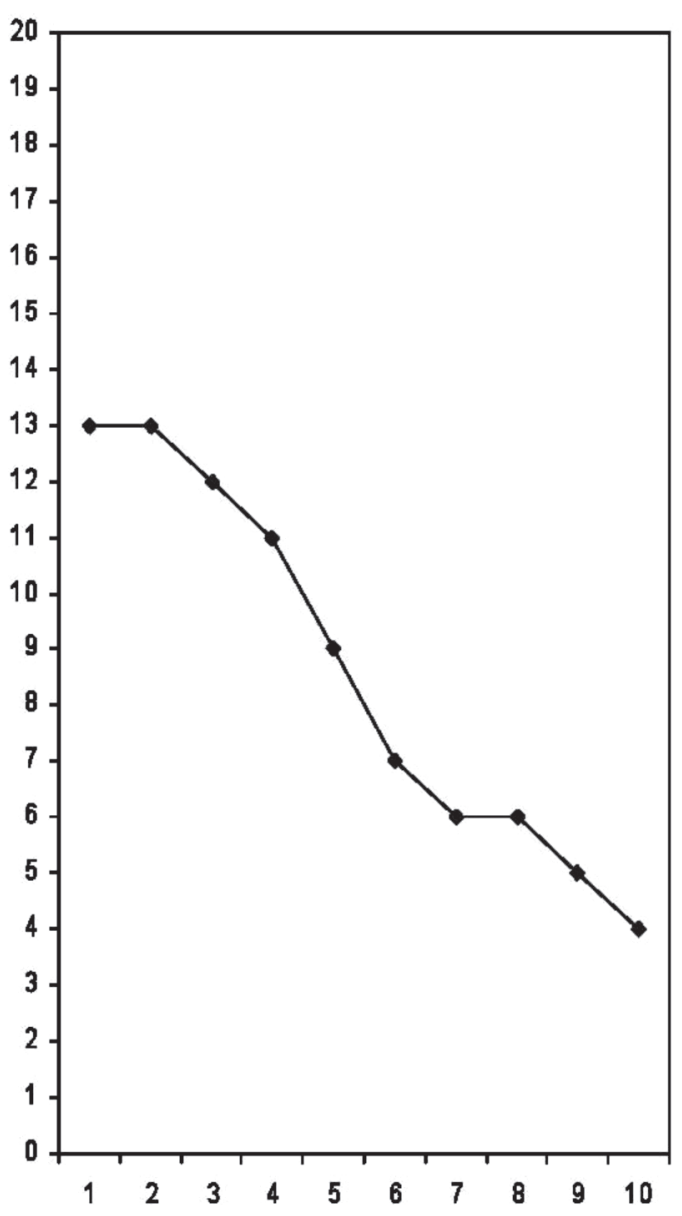

Figura 1. Puntuaciones en la CDSS.

En cuanto a los datos en la PANSS al concluir las 10 semanas de tratamiento se observa una disminución de 20 puntos en el resultado global. Concretamente, se ha producido una significativa disminución de la psicopatología general (13 puntos) que presentaba el paciente al comienzo de la intervención. El resultado en la escala de Depresión confirma los resultados obtenidos en la CDSS respecto a la sintomatología depresiva al pasar de 5 puntos a 1 punto. Tras ésta, las escalas en la que más reducción se produce son las de Sentimientos de culpa y Evitación social activa donde se obtiene una reducción de dos puntos (de 3 a 1 y 4 a 2 , respectivamente), siendo dos escalas que miden aspectos que pueden guardar relación 
Tabla 2

Puntuaciones en las medidas de tratamiento

\begin{tabular}{lccc}
\hline & Pretratamiento & Postratamiento & Seguimiento \\
\hline CDSS & 13 & 4 & 3 \\
PANSS-Positivo & 11 & 9 & 8 \\
PANSS-Negativo & 22 & 17 & 15 \\
PANSS-Psicop. General & 37 & 24 & 20 \\
PANSS-TOTAL & 70 & 50 & 43 \\
EROS & 17 & 25 & 24 \\
\hline
\end{tabular}

Nota. CDSS = Calgary Depression Scale for Schizophrenia. PANSS $=$ The Positive and Negative Sindrome Scale. EROS = Environmental Reward Observation Scale.

con el padecimiento de depresión. Se han producido descensos en las escalas de Ansiedad, Preocupación y Tensión motora desapareciendo los Contenidos inusuales del pensamiento. En lo concerniente a la puntuación en las escalas que valoran la sintomatología propiamente psicótica (PANSS-P y PANSS-N) se detectan cierta mejora en aspectos como la sintomatología psicótica de tipo positivo ( 2 puntos) y negativo (4 puntos) en particular en la escala de Retraimiento social (de 5 a 3 puntos).

Los resultados se mantienen y acrecientan tras el seguimiento. Respecto a la sintomatología depresiva el paciente se mantiene asintomático, presentando una puntuación 3 puntos. En cuanto a los datos de la PANSS se detecta un descenso en la puntuación global de 7 puntos (de 50 a 43 puntos), con disminuciones de 2 puntos en la sintomatología negativa (de 17 a 15 puntos), y de 1 punto (Delirios) en sintomatología positiva. Es en las escalas de psicopatología general (PANSS-PG) donde se vuelve a observar el mayor decremento con una mejoría de 4 puntos en el seguimiento (de 24 a 20 puntos) sin observarse intensificación en ninguna de las escalas. En cuento a los va- lores específicos se confirma, a través de la escala Depresión, los datos obtenidos en el seguimiento respecto a la sintomatología depresiva recogidos en la CDSS indicándose, en ambas, ausencia de criterios para el diagnóstico de un síndrome depresivo. En cuanto a otras escalas, como la de Tensión motora y Atención deficiente, el paciente deja encontrarse afectado como indica la puntuación de 1 en las dos escalas. Finalmente resaltar que tanto en la escala Preocupación como en la de Trastornos de la volición se muestran un nuevo descenso (de 3 a 2 puntos).

Finalmente, respecto a la escala EROS la puntuación en la valoración inicial fue de 17 puntos, a las diez semanas se obtuvo una puntuación de 25 , y en el seguimiento de 24 puntos (los datos de la adaptación española indican una media de 22,89 y una desviación tipo de 5,95 puntos en la población clínica).

\section{Discusión}

En el presente artículo se ha expuesto la intervención aplicada para el tratamiento de la sintomatología depresiva en un paciente diagnosticado de esquizofrenia paranoide. 
La intervención consistió en 10 semanas de tratamiento de activación conductual para la depresión. El paciente presentó desde el principio una importante motivación y buen seguimiento del plan terapéutico concluyendo todo el programa previsto al inicio. Deseaba acabar con su nivel de escasa actividad social y lúdica. Consideraba, en concordancia con lo trabajado en las primeras entrevistas sobre el modelo de activación conductual, que era un factor decisivo en su estado de ánimo el abandono de actividades y que su recuperación paulatina iba a suponer una mejora anímica. Estuvo de acuerdo desde el principio en el modelo de trabajo planteado. En todo momento se contó con su opinión aspecto que fue recibido con gran agrado ya que contrastaba con la actitud recibida por gran parte de los profesionales que le habían atendido hasta ese momento. De esta forma él se convertía en el protagonista principal de su tratamiento y no en un mero receptor de éste. Lo anterior resultó en un incremento de su nivel motivacional y su sensación de control sobre lo que le ocurría en el día a día.

Los resultados demuestran que la intervención ha resultado muy eficaz para disminuir la sintomatología depresiva inicial dejando de cumplir criterios de episodio depresivo al finalizar el tratamiento y manteniéndose este resultado durante el seguimiento, confirmado tanto por la CDSS como por la escala de depresión de la PANSS. Queda por dilucidar si el añadir el enfoque de la Terapia de Activación Conductual puede incrementar la eficacia de las intervenciones tradicionales en rehabilitación psicosocial en lo que respecto a la sintomato- logía negativa y positiva, propia del trastorno esquizofrénico, o su eficacia se queda reducida a la sintomatología depresiva. En éste caso sí se observa una disminución de la sintomatología general y, en algunos aspectos, de la psicopatología propiamente psicótica. Que esto se deba a un efecto directo del tratamiento o como resultado secundario de la disminución de la sintomatología depresiva podría ser tema de futuros estudios.

La disminución de la sintomatología depresiva se ha producido al mismo ritmo que se incrementaba el número de actividades que iba realizando el paciente. Unido a ello, como bien se refleja en los resultados en la escala EROS, se detecta un incremento en la percepción que tiene el paciente del reforzamiento positivo proveniente del medio. Permite que el paciente sea más consciente de cómo su actuación influye en las consecuencias que obtiene del medio incrementando su capacidad para obtener refuerzo del contexto y prevenir posibles recaídas futuras. Unido a esto, el paciente establecía un vínculo entre el incremento del tiempo que dedicaba a actividades valiosas para él y una mejoría en la relación con su madre.

En las últimas décadas se ha ido desarrollando una perspectiva contextual para el abordaje de los distintos trastornos psicológicos. De especial importancia han supuesto los estudios sobre la eficacia del componente conductual de la terapia cognitiva en la depresión (Jacobson et al., 1996) y los estudios posteriores que han confirmado y extendido las conclusiones iniciales (Dimidjian et al., 2006). Consideramos que se abre, de éste modo, nuevos caminos, tanto teóricos como prácticos, que pueden su- 
poner grandes avances en los próximos años para la psicología clínica.

La filosofía que subyace al proceso de rehabilitación en salud mental supone que el paciente vaya asumiendo y recuperando las capacidades previas o, en caso de su ausencia, su aprendizaje (Rodríguez, 1997). Implica el desarrollo de actividades paulatinas tras el estudio individual de cada caso. Se estima que la Activación Conductual comparte grandes similitudes con la práctica cotidiana que se realiza en las unidades de rehabilitación de salud mental por lo que es un enfoque teórico que puede enriquecer la labor de los profesionales que atienden a pacientes con trastornos psicóticos crónicos. A nivel práctico, se puede considerar que el tratamiento de activación conductual se muestra como una opción terapéutica válida para su aplicación en pacientes con trastornos mentales graves que presentan sintomatología depresiva.

Se espera que el presente artículo anime a otros profesionales a aplicar la Terapia de Activación Conductual al área de los trastornos mentales graves y confirmar con un número mayor de estudios nuestros resultados iniciales.

\section{Referencias}

Addington, D. Addington, J., \& Schissel B. (1990). A depression rating scale for schizophrenics. Schizophrenia Reseach, 3, 247-251.

Armento, M. E. A. \& Hopko, D. R. (2007). The Environmental Reward Observation

Scale (EROS): Development, validity, and reliability. Behavior Therapy, 38, 107-119.

Barraca Mairal, J. ( 2009). La Activación Conductual (AC) y la Terapia de Activación
Conductual para la Depresión (TACD). Dos protocolos de tratamiento desde el modelo de la activación conductual. EduPsykhé. Revista de Psicología y Educación, 8, 23-50.

Barraca, J. \& Pérez-Álvarez, M. (2010). Adaptación española del Environmental Reward Observation Scale (EROS). Ansiedad y Estrés, 16, 95-107.

Daughters, S. B., Braun, A. R., Sargeant, M. N., Reynolds, E.K.,Hopko, D.R., Blanco, C., Nunes, E., \& Lejuez, C. W. (2008). Effectiveness of a brief behavioral treatment for inner-city illicit drug users with elevated depressive symptoms: The Life Enhancement Treatment for Substance Use (LETS ACT!). Journal of Clinical Psychiatry, 69, 122-129.

Dimidjian, S., Hollon, S. D., Dobson, K. S., Schmeling, K. B., Kohlenberg, R. J., Addis, et al. (2006), Randomized trial of Behavioral Activation, Cognitive Therapy and antidepressant medication in the acute treatment of adult. Journal of Consulting and Clinical Psychology, 74, 658-670.

Elkin, I., Shea, M. T., Watkins, J. T., Imber, S. S., Sotsky, S. M., Collins, J. F.,... Parloff, M. B. (1989). Treatment of Depression Collaborative Reseach Program. General Effectiveness of treatments. N.I.M.H. Archives of General Psychiatry, 46, 973-982.

Herrnstein, R. J. (1970). On the law of effect. Journal of Experimental Analysis of behavior. 13, 243-266.

Hopko, D. R., Bell, J. L., Armento, M. E. A., Hunt, M. K., \& Lejuez, C. W. (2005). Behavior therapy for depressed cancer patients in primary care. Psychotherapy: Theory, Research, Practice, Training, 42, 236-243.

Hopko, D. R., Robertson, S. M. C., \& Lejuez, C. W. (2006). Behavioral Activation for anxiety disorders. The Behavior Analyst Today, 7, 212- 232.

Hopko, D. R., Sanchez, L., Hopko, S. D., Dvir, S., \& Lejuez, C. W. (2003). Behavioral activation and the prevention of suicidal behaviours in patients with borderline personality 
disorder. Journal of Personality Disorders, 17, 460-478.

Jacobson, N. S., Dobson, K. S., Truax, P. A., Addis, M. E., Koerner, K., Gollan, J. K.,... Prince, S. E. (1996). A component analysis of cognitivebehavioral treatment for depression. Journal of Consulting and Clinical Psychology 64, 295-304.

Jacobson, N. S., Martell, C. R., \& Dimidjian, S. (2001). Behavioral activation treatment for depression: Returning to contextual roots. Clinical Psychology: Science and Practice, 8, 255-270.

Kay S. R., Fiszbein, A, \& Opler, L. A. (1987). The positive and negative syndrome scale (PANSS) for schizophrenia. Schizophrenia Bulletin, 13, 261-276.

Lejuez, C. W., Hopko, D. R., \& Hopko, S. D. (2001). A brief Behavioral Activation treatment for depression: Treatment manual. Behavior Modification, 25, 225-286.

Martell, C. R., Addis, M. E., \& Jacobson, N. S. (2001). Depression in context: Strategies for guided action. New York: W. W. Norton.

Meltzer H.Y.\&Baldessarini R. J. (2003). Reducing the risk for suicide in schizophrenia and affective disorders. Journal of Clinical Psychiatry, 64, 1122-1129.

NICE (2004). Depression: Management of depression in primary and secondary care: National clinical practice guideline, number 23.

Organización Mundial de la Salud. (1992). CIE-10. Trastornos Mentales y del Comportamiento. Décima Revisión de la Clasificación Internacional de las Enfermedades. Descripciones Clínicas \& pautas para el diagnóstico. Ginebra: Autor.

Overall, J. E. \& Gorham, D. R. (1962). The Brief Psychiatric Rating Scale. Psychological Reports, 10, 799-912.

Pérez Álvarez, M. (2006). La terapia de conducta de tercera generación. EduPsykhé: Revista de Psicología y Psicopedagogía, 5, 159-172.
Peralta V. \& Cuesta M. J. (1994). Validación de la escala de los síndromes positivo y negativo (PANSS) en una muestra de esquizofrénicos españoles. Actas Luso-Españolas de Neurología y Psiquiatría. 22, 171-177.

Rodríguez, A. (Coord.) (1997). Rehabilitación psicosocial de personas con trastornos mentales crónicos. Madrid: Editorial Pirámide.

Ruggiero, K. J., Morris, T. L., Hopko, D. R., \& Lejuez, C. W. (2007). Application of behavioral activation treatment for depression to an adolescent with a history of child maltreatment. Clinical Case Studies, 6, 64-78.

Thase,M.E., Greenhouse,J.B.,Frank, E., Reynolds, C. F., Pilkonis, P. A., Hurley, K.,... Kupfer D. J. (1997). Treatment of major depression with psychotherapy or psychotherapypharmacotherapy combinations. Archive of General Psychiatry, 54, 1009-1015. 
